\section{Complete closure of a large gastric perforation caused by percutaneous endoscopic gastrostomy using over-the-scope clips}

Complications of percutaneous endoscopic gastrostomy (PEG) include bleeding, infection, miss-puncture of other organs, and perforation $[1,2]$. Herein we report on a large gastric perforation resulting from a PEG tube insertion that was successfully treated with a double over-the-scope clip technique.

An 84-year-old woman with a history of cerebral infarction and dysphagia was submitted for PEG gastropexy placement to guarantee enteral nutrition. Three point fixations of the abdominal wall and stomach had been performed under fluoroscopic assistance, before the PEG damper (Ideal PEG kit; Olympus, Tokyo, Japan) was inserted into stomach. While fluoroscopy was being performed to confirm the correct placement of the PEG damper, a massive amount of free air became evident ( $\bullet$ Fig.1). A large lacerated wound with an opening of $3 \mathrm{~cm}$ in diameter was identified next to the damper, and the PEG tube trajectory through the abdominal wall and peritoneum could be seen ( $\bullet$ Fig. 2 a).

The pneumoperitoneum was treated by inserting a needle (Surgineedle, $120 \mathrm{~mm}$,
14G; Covidien Japan, Tokyo, Japan) to deflate the air. The perforation was then closed using an over-the-scope clip (OTSC, type GC, $10 \mathrm{~mm}$ in diameter; Ovesco, Tübingen, Germany) ( $\bullet$ Video 1 ). After grasping both edges of the full-thickness perforation site using a Twin grasper, OTSC closure was accomplished using two clips ( $\bullet$ Fig. 2 b). The second OTSC served to close the gap between the previous OTSC and the PEG tube ( $\bullet$ Fig. 2 c). After the wound had been closed, fluoroscopy showed the disappearance of the pneumoperitoneum ( $\bullet$ Fig.3) and an endoscopic retroflexed view revealed the hermetically closed defect ( Fig.4). Enteral nutrition using the PEG was started 3 days after the closure, without any complications.

This report shows that it was possible to avoid surgery by the use of an advanced endoscopic repair technique.

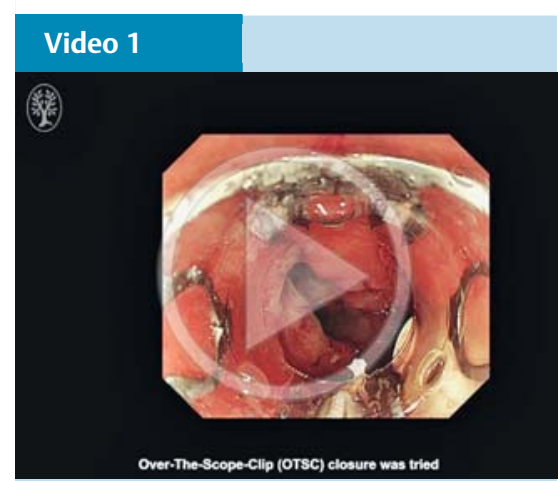

Fluoroscopic image showing the pneumoperitoneum; endoscopic views of the perforation and its closure using a Twin grasper and two over-the-scope clips (OTSCs); confirmation of closure is shown on fluoroscopy by the disappearance of the pneumoperitoneum and the stomach filled with insufflated air.

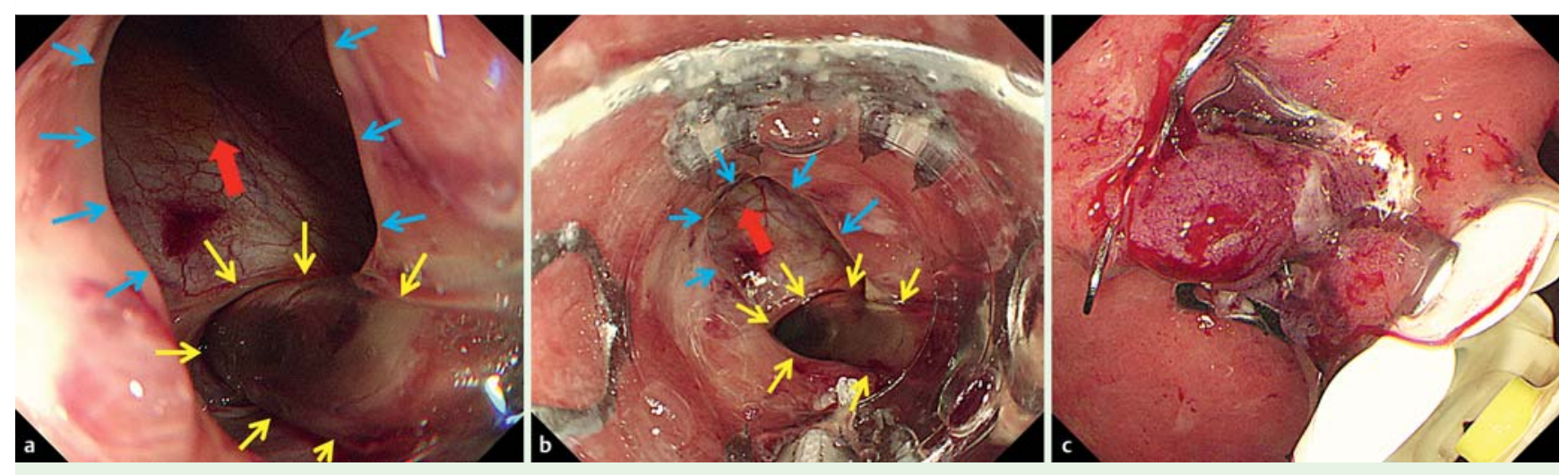

Fig. 2 Endoscopic views showing: a a large perforation of $3 \mathrm{~cm}$ in diameter (blue arrows) next to the percutaneous endoscopic gastrostomy (PEG) damper, with the PEG tube visible through the abdominal wall (yellow arrows) and peritoneum (red arrow); b closure of the perforation (blue arrows) using an over-thescope clip (OTSC); $\mathbf{c}$ a second OTSC that was applied to close the gap between the first OTSC and the PEG tube. 


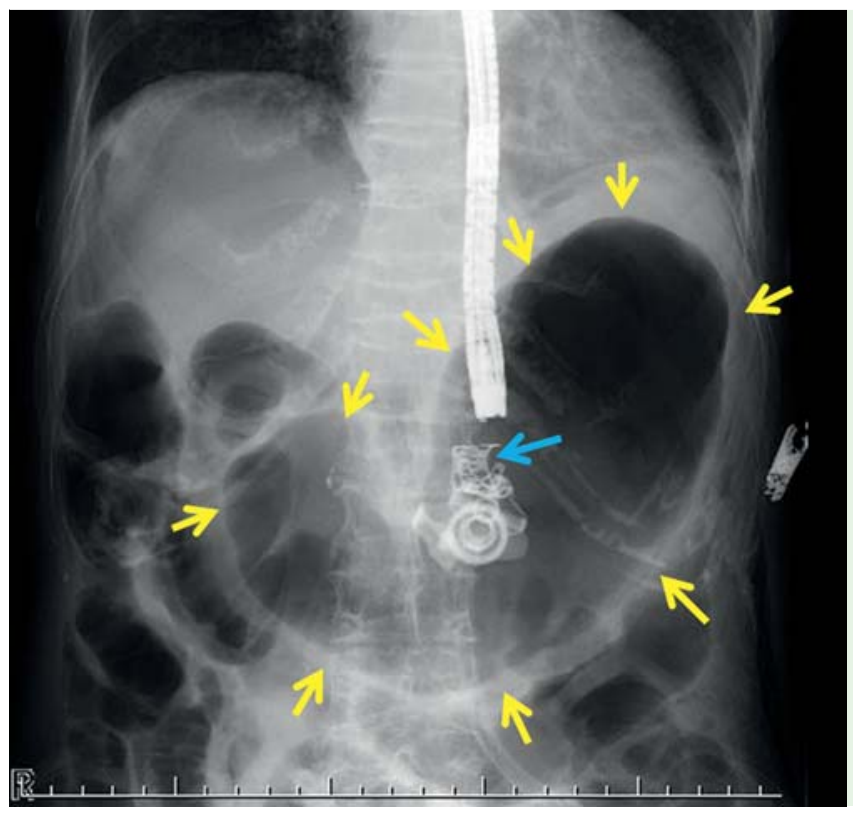

Fig. 3 Fluoroscopic view after closure with two over-thescope clips (OTSCs; blue arrows) showing the disappearance of the pneumoperitoneum and the stomach filled with insufflated air (yellow arrows).
Endoscopy_UCTN_Code_CPL_1AH_2AI

\section{Competing interests: None}

\section{Hirohito Mori, Hideki Kobara, Shintaro Fujihara, Noriko Nishiyama, Taiga Chiyo, Tsutomu Masaki}

Department of Gastroenterology and Neurology, Kagawa University, Kagawa, Japan

\section{References}

1 Cagin YF, Atayan Y, Erdoğan MA et al. Relationship of percutaneous endoscopic gastrostomy-related mortality and morbidity rates and effectiveness with advancing age. Acta Gastroenterol Belg 2015; 78: 292 - 298

2 Rahnemai-Azar AA, Rahnemaiazar AA, Naghshizadian $R$ et al. Percutaneous endoscopic gastrostomy: indications, technique, complications and management. World J Gastroenterol 2014; 20: 7739-7751

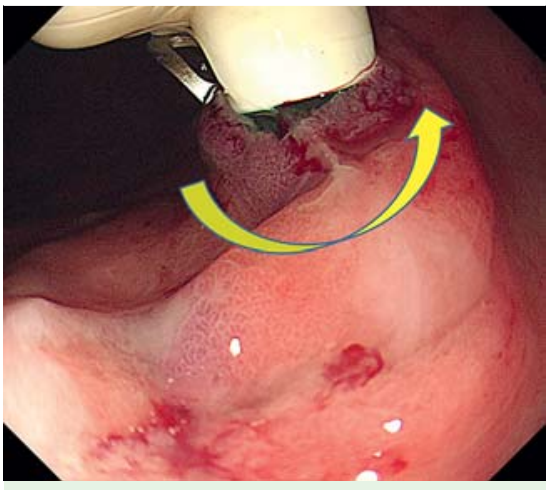

Fig. 4 Retroflexed endoscopic view showing hermetic closure of the perforation (yellow curved arrow).
Bibliography

DOI http://dx.doi.org/

10.1055/s-0035-1569653

Endoscopy 2016; 48: E8-E9

(c) Georg Thieme Verlag KG

Stuttgart · New York

ISSN 0013-726X

\section{Corresponding author}

\section{Hirohito Mori, MD, PhD}

Department of Gastroenterology and Neurology Kagawa University

1750-1 Ikenobe

Miki, Kita

Kagawa 761-0793

Japan

Fax: +81-87-8912158

hiro4884@med.kagawa-u.ac.jp 